\title{
Two-loop applications of the program SecDec
}

\section{Sophia Borowka*}

Max Planck Institute for Physics, Föhringer Ring 6, 80805 Munich, Germany

E-mail: sborowka@mpp.mpg.de

\section{Gudrun Heinrich}

Max Planck Institute for Physics, Föhringer Ring 6, 80805 Munich, Germany

E-mail: gudrun@mpp.mpg.de

SECDEC is a program which can be used for the factorisation of poles and subsequent numerical evaluation of multi-loop integrals, in particular massive two-loop integrals. We show applications to two-loop master integrals entering the calculation of top quark pair production at NNLO, and to the dominant momentum dependent two-loop corrections to the neutral Higgs boson masses in the MSSM.

11th International Symposium on Radiative Corrections (Applications of Quantum Field Theory to Phenomenology) (RADCOR 2013),

22-27 September 2013

Lumley Castle Hotel, Durham, UK

${ }^{*}$ Speaker. 


\section{Introduction}

Precision measurements are of primary importance in order to find deviations from the Standard Model and to identify their origin as "New Physics". Certainly, this is only possible in conjunction with precise theory predictions, requiring the calculation of higher order corrections. While the calculation of corrections at next-to-leading order has reached an impressive level of automation meanwhile, corrections beyond one loop still require quite some efforts both on the conceptual and on the technical side before they can be produced in a largely automated way.

An important ingredient for the calculation of higher order corrections are the loop integrals entering the virtual corrections. Analytical expressions for integrals beyond one loop are only known for integrals depending on rather few mass scales. Therefore, as soon as several mass scales are involved, numerical methods to calculate these integrals are often indispensable. However, the latter can only be applied after extraction of possible ultraviolet and/or infrared poles contained in the diagrams.

The program SECDEC [1-3], performs the task of isolating dimensionally regulated singularities in an automated way, based on the algorithm of sector decomposition [4-6]. Other implementations of sector decomposition into public programs are also available [7-9]. However, the latter are more or less restricted to the Euclidean region, while SECDEC can deal with physical kinematics, including for instance mass thresholds.

In these proceedings, applications of the program SECDEC 2.1 to massive two-loop integrals will be presented.

\section{Structure of the program}

The program can be divided into two main branches, one for the computation of loop integrals, the other one for the treatment of more general parametric functions (corresponding to the directories loop and general). A flowchart of the program is depicted in Fig. 1. For a detailed description of the program we refer to [1-3], here we will only mention the main aspects, and describe features not highlighted previously.

The loop part has been extended in version 2.1 to be able to treat parametric integrals which are not in the canonical form as obtained directly from standard Feynman parametrisation. The integrals can have a different format, coming for example from variable transformations and/or analytical integrations over some of the Feynman parameters. Hence, as these functions differ from the standard representation which the program would derive from the propagators in an automated way, they have to be defined in an input file by the user. Contour deformation is available for these functions, as they are assumed to originate from a Feynman integral structure, where poles on the real axis are protected by the infinitesimal $i \delta$ prescription.

The setup in the directory general is designed to deal with even more general parametric functions, where the integrand can consist of a product of arbitrary length of polynomial functions to some power. However, these functions should have only endpoint singularities (i.e. dimensionally regulated singularities at the integration boundaries). Contour deformation is not available in this case because the correct sign of the imaginary part for the deformation into the complex plane cannot be inferred if the assumption of an underlying Feynman integral structure is dropped. 
The procedure of iterated sector decomposition and subsequent numerical integration is the same for all the different types of input functions, and is described in $[1,2,10]$.

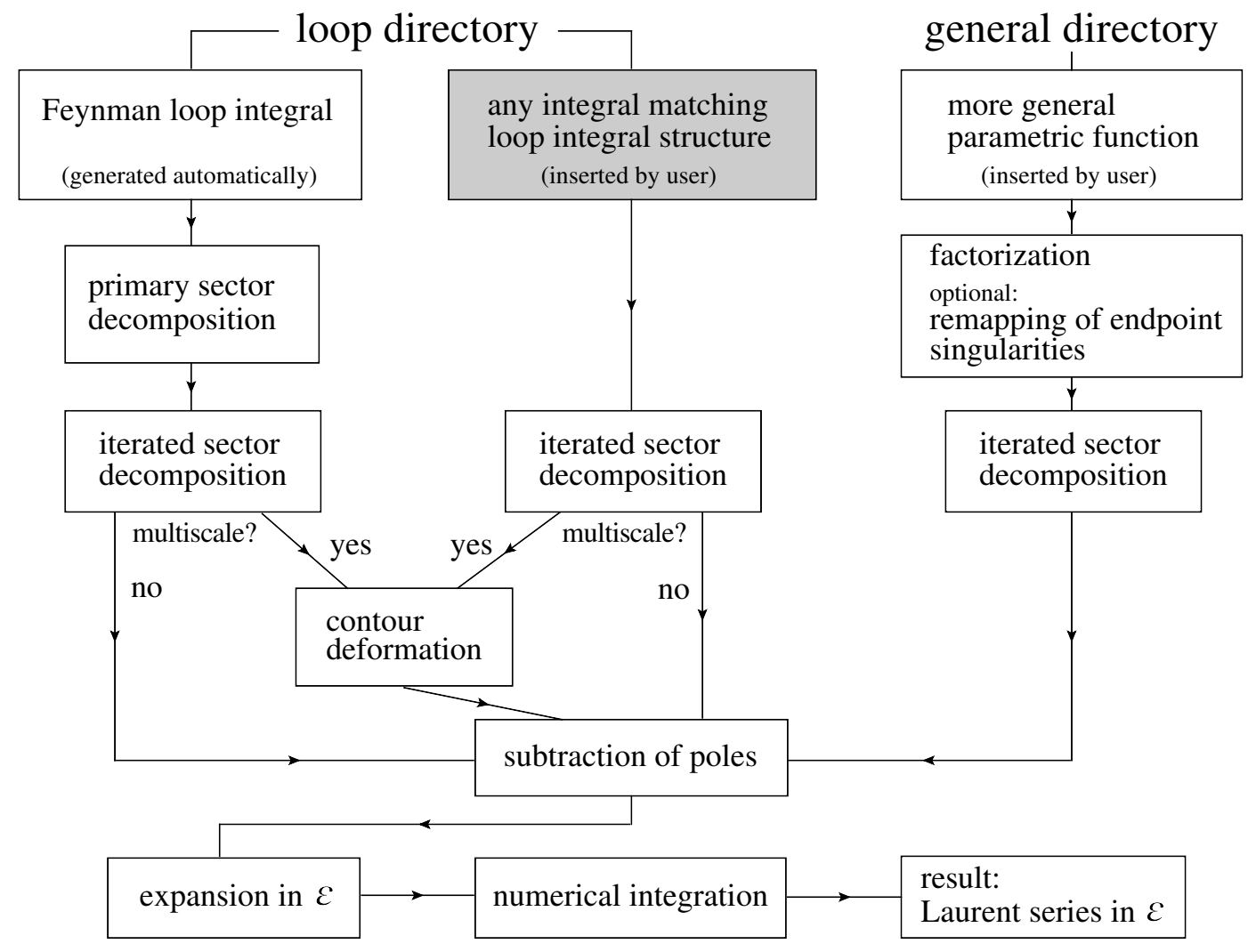

Figure 1: Flowchart showing the structure of the program SECDEC.

The current version 2.1.4 of the program can be downloaded from

http://projects.hepforge.org/secdec.

Unpacking the tar archive will create a directory called SecDec-2.1.4. Changing to this directory and running./install will compile the CUBA library [11] needed for the numerical integration. Prerequisites are Mathematica (version $\geq 6$ ), Perl (installed by default on most Unix/Linux systems) and a C++ compiler, respectively a Fortran compiler if the Fortran option is used.

More details about the usage, in particular about the option userdefined, can be found in Refs. [1,3], and also in the documentation coming with the program.

\section{Applications}

\subsection{Non-planar massive two-loop diagrams entering NNLO $t \bar{t}$ production}

The most complicated master topologies occurring in the two-loop corrections to $t \bar{t}$ production in the $g g$ channel are the non-planar seven-propagator integrals shown in Fig. 2.

Analytic results for the integral containing a sub-diagram with a massive loop (called ggttl here), are not available, while analytic results for the diagram corresponding to massless fermionic corrections in a sub-loop (called ggtt2 here) have become available very recently [12]. However, 


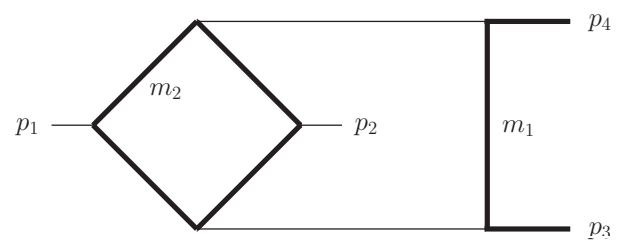

(a) ggtt1

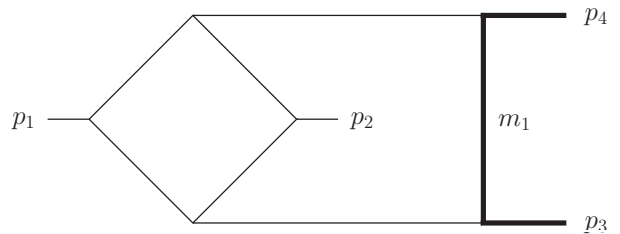

(b) ggtt2

Figure 2: Massive non-planar two-loop box diagrams entering the heavy (a) and light (b) fermionic correction to the $g g \rightarrow t \bar{t}$ channel; the bold lines denote massive particles.

the numerical evaluation of $g g t t l$ with SECDEC is much easier than the one of $g g t t 2$, due to its less complicated infrared singularity structure. While the leading poles of ggtt 2 are of order $\mathscr{O}\left(\varepsilon^{-4}\right)$, and intermediate expressions during sector decomposition contain (spurious) poles where the degree of divergence is higher than logarithmic, the integral ggttl is finite and free from spurious singularities. Therefore we can evaluate ggttl with SECDEC 2.1 using the fully automated setup. In contrast, for ggtt 2 it turned out to be advantageous to make some analytical manipulations beforehand. In particular, it was useful to perform one parameter integration analytically before feeding the integral into the decomposition and numerical integration algorithm. Further, we introduced special transformations to reduce the occurrence of spurious singularities, which are described in detail in [3,13]. These manipulations lead to functions which were not in the "standard form" of Feynman parameterised loop integrals anymore. This entailed the development of a setup to treat "non-standard" parametric functions mentioned in Section 2, which has been made available for the user, as it can be beneficial in similar contexts.

Numerical results for the ggtt2 diagram are shown in Fig. 3, where we used the numerical values $p_{3}^{2}=p_{4}^{2}=m^{2}=1, s_{23}=-1.25, s_{13}=2 m^{2}-s_{12}-s_{23}$, and we extract an overall factor of $-16 \Gamma(1+\varepsilon)^{2}$. We only show results for the finite part here, as it is the most complicated one and therefore more interesting than the pole coefficients.

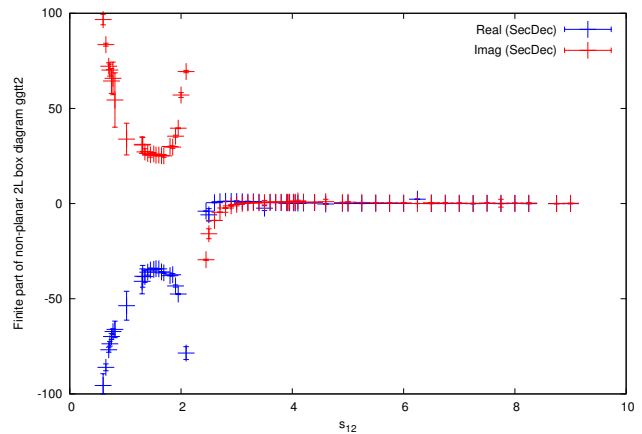

(a) finite part, including threshold

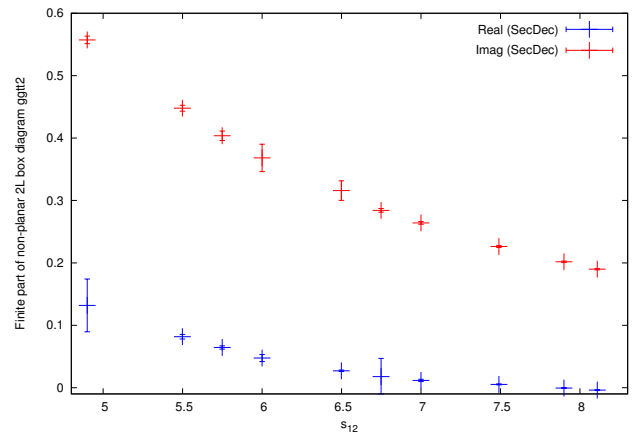

(b) finite part, beyond threshold

Figure 3: Results for the finite part of the scalar integral ggtt2, (a) for a larger kinematic range, (b) zoom into a region further away from threshold. The vertical bars denote the numerical integration errors.

Numerical results for the diagram ggttl are shown in Fig. 4 for both the scalar integral and an irreducible rank two tensor integral. 


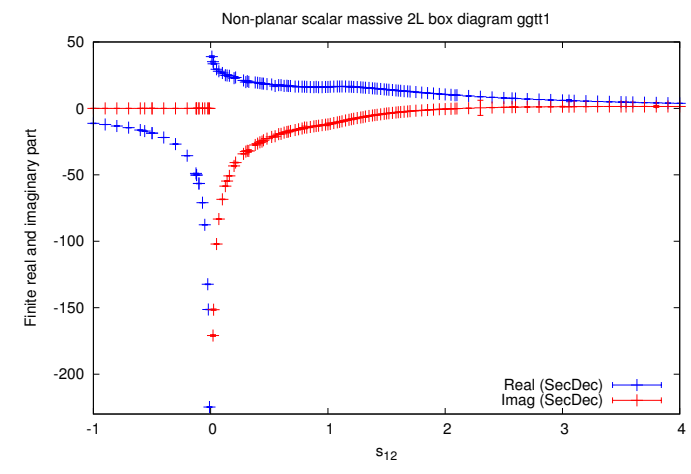

(a) scalar integral

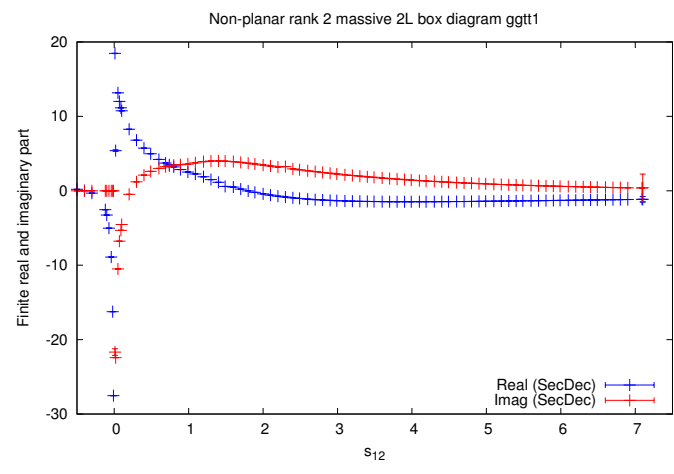

(b) rank 2 tensor integral

Figure 4: Results for the scalar integral ggttl and the corresponding rank two tensor integral with $k_{1} \cdot k_{2}$ in the numerator. We vary $s_{12}$ and fix $s_{23}=-1.25, m_{2}=m_{1}, p_{3}^{2}=p_{4}^{2}=m_{1}^{2}=1$.

For the results shown in Fig. 4 we used the numerical values $m_{1}^{2}=m_{2}^{2}=m^{2}=1, s_{23}=$ $-1.25, s_{13}=2 m^{2}-s_{12}-s_{23}$. We set $m_{1}^{2}=m_{2}^{2}$ because this is the only case occurring in the process $g g \rightarrow t \bar{t}$ at two loops if the $b$-quarks are assumed to be massless. However, we also verified that the case $m_{1}^{2} \neq m_{2}^{2}$ can be evaluated without a significant increase in computing time compared to the equal mass case.

The timings for one kinematic point for the scalar integral in Fig. 4(a) range from 11-60 secs for points far from threshold to $1.6 \times 10^{3}$ seconds for a point very close to threshold, with an average of about 500 secs for points in the vicinity of the threshold. A relative accuracy of $10^{-3}$ has been required for the numerical integration, while the absolute accuracy has been set to $10^{-5}$. For the tensor integral, the timings are better than in the scalar case, as the numerator function present in this case smoothes out the singularity structure. The timings were obtained on a single machine using Intel i7 processors and 8 cores.

\subsection{Corrections to the neutral CP even Higgs bosons in the MSSM}

The Higgs sector of the MSSM consists of two doublets $H_{1}$ and $H_{2}$, which can be written as

$$
H_{1}=\left(\begin{array}{c}
H_{1}^{0} \\
H_{1}^{-}
\end{array}\right)=\left(\begin{array}{c}
v_{1}+\frac{1}{\sqrt{2}}\left(\phi_{1}^{0}+\mathrm{i} \chi_{1}^{0}\right) \\
-\phi_{1}^{-}
\end{array}\right), \quad H_{2}=\left(\begin{array}{c}
H_{2}^{+} \\
H_{2}^{0}
\end{array}\right)=\left(\begin{array}{c}
\phi_{2}^{+} \\
v_{2}+\frac{1}{\sqrt{2}}\left(\phi_{2}^{0}+\mathrm{i} \chi_{2}^{0}\right)
\end{array}\right) .
$$

The vacuum expectation values $v_{1}$ and $v_{2}$ define the angle $\tan \beta=v_{2} / v_{1}$. At tree level, the mass matrix of the neutral CP-even Higgs bosons in the $\phi_{1}, \phi_{2}$ basis can be written as

$$
M_{\text {Higgs }}^{2 \text {,tree }}=\left(\begin{array}{cc}
M_{A}^{2} \sin ^{2} \beta+M_{Z}^{2} \cos ^{2} \beta & -\left(M_{A}^{2}+M_{Z}^{2}\right) \sin \beta \cos \beta \\
-\left(M_{A}^{2}+M_{Z}^{2}\right) \sin \beta \cos \beta & M_{A}^{2} \cos ^{2} \beta+M_{Z}^{2} \sin ^{2} \beta
\end{array}\right),
$$

where $M_{A}$ is the mass of the CP-odd neutral Higgs boson $A$. The rotation to the basis formed by the Higgs bosons $H^{0}, h^{0}$ is given by

$$
\left(\begin{array}{c}
H^{0} \\
h^{0}
\end{array}\right)=\left(\begin{array}{cc}
\cos \alpha & \sin \alpha \\
-\sin \alpha & \cos \alpha
\end{array}\right)\left(\begin{array}{l}
\phi_{1}^{0} \\
\phi_{2}^{0}
\end{array}\right)
$$


where

$$
\tan (2 \alpha)=\tan (2 \beta) \frac{M_{A}^{2}+M_{Z}^{2}}{M_{A}^{2}-M_{Z}^{2}}, \quad-\frac{\pi}{2}<\alpha<0
$$

The need for higher order corrections to the MSSM Higgs boson masses is obvious from the fact that at tree level, the mass of light CP-even Higgs boson is bound from above through the relation $m_{h} \leq \min \left(M_{Z}, M_{A}\right)|\cos (2 \beta)|$.

The status of currently available two-loop self-energy corrections to the Higgs boson masses in the MSSM with real parameters (rMSSM) is the following. The corrections $\mathscr{O}\left(\alpha_{s} \alpha_{t}\right)$ [14-19], $\mathscr{O}\left(\alpha_{t}^{2}\right)$ [14, 20,21], $\mathscr{O}\left(\alpha_{s} \alpha_{b}\right)$ [22], $\mathscr{O}\left(\alpha_{t} \alpha_{b}\right)$ [23], $\mathscr{O}\left(\alpha_{b}^{2}\right)$ [23] are known in the gaugeless limit (i.e. vanishing gauge couplings to the SM vector bosons), and using the approximation $p^{2}=0$ for the external momentum of the self-energies. The strong coupling constant is denoted by $\alpha_{s}$ as usual, while $\alpha_{\{t, b\}}$ denote the Yukawa couplings of the top (bottom) quarks, where $\alpha_{\{t, b\}}=$ $y_{\{t, b\}}^{2} /(4 \pi)$. At three loops, the $\mathscr{O}\left(\alpha_{s}^{2} \alpha_{t}\right)$ corrections are known [24-26] in the gaugeless limit and in the $p^{2}=0$ approximation. The largest uncertainties on the presently known results stem from the contributions of the momentum dependent self-energy corrections at two loop order. The latter have been calculated in $[27,28]$ based on an effective potential approach within the $\overline{D R}$ scheme. However, in order to incorporate the corrections into the public program FeynHiggs $[29,30]$, it is more convenient to use on-shell renormalisation for all the masses. To this aim, we computed the self-energies $\Sigma_{\phi_{i} \phi_{j}}\left(p^{2}\right)$ at two loops at $\mathscr{O}\left(\alpha_{s} \alpha_{t}\right)$, keeping the full momentum dependence. We use the $\overline{D R}$ scheme for the field renormalisation, and on-shell renormalisation for all other renormalisation constants, as explained e.g. in [17].

The reduction of the amplitude to scalar master integrals is performed with the help of the package TwoCalc [31] for the two-loop diagrams, while the one-loop counter term diagrams are reduced using FormCalc [32]. The two-loop topologies for which a full analytical result is not available, shown in Fig.5, are calculated numerically with SECDEC. In total, these amount

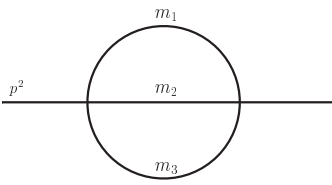

(a) $\mathrm{T} 234$

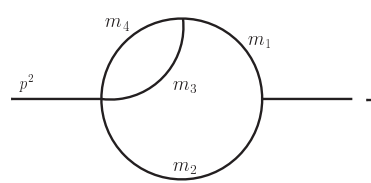

(b) T1234

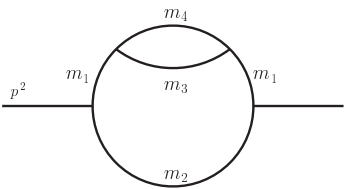

(c) $\mathrm{T} 11234$

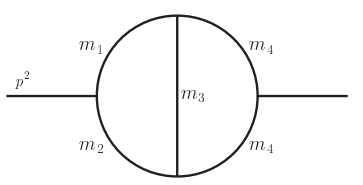

(d) $\mathrm{T} 12345$

Figure 5: Two-loop two-point topologies treated with SECDEC with up to four different mass scales

to 37 different mass configurations, where the two-loop diagrams can have up to four different masses. We tested the numerical integration with SECDEC for integrals where the kinematic values differed by up to 14 orders of magnitude. The timings range between 0.01 secs and 100 secs with an achieved relative accuracy of $10^{-5}$ to $10^{-11}$. Two representative results are shown in Fig. 6. The configuration in Fig. 6(a) has three different mass scales where we set $m_{1}=m_{2}=173.2$ $\mathrm{GeV}, m_{3}=826.8 \mathrm{GeV}$ and $m_{4}=1.5 \mathrm{TeV}$. In Fig. $6(\mathrm{~b})$, the numerical values $m_{1}=1173.2 \mathrm{GeV}$, $m_{2}=826.8 \mathrm{GeV}, m_{3}=1.5 \mathrm{TeV}$ and $m_{4}=173.2 \mathrm{GeV}$ are chosen.

More detailed results will be given elsewhere [33]. 


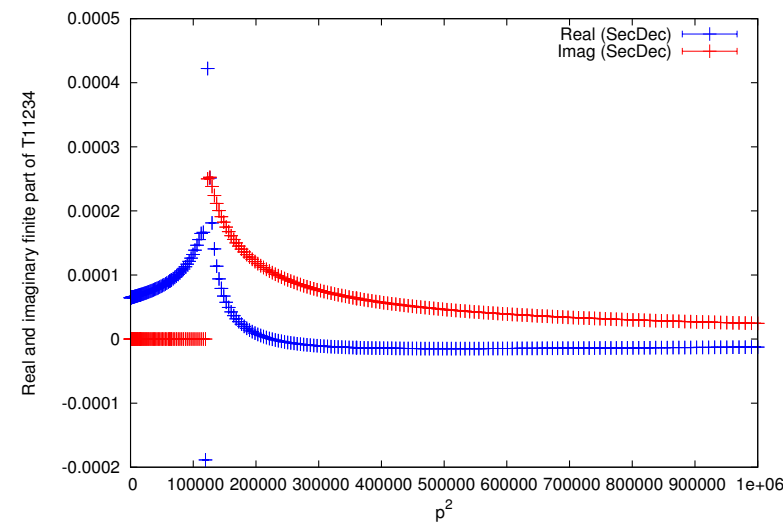

(a)

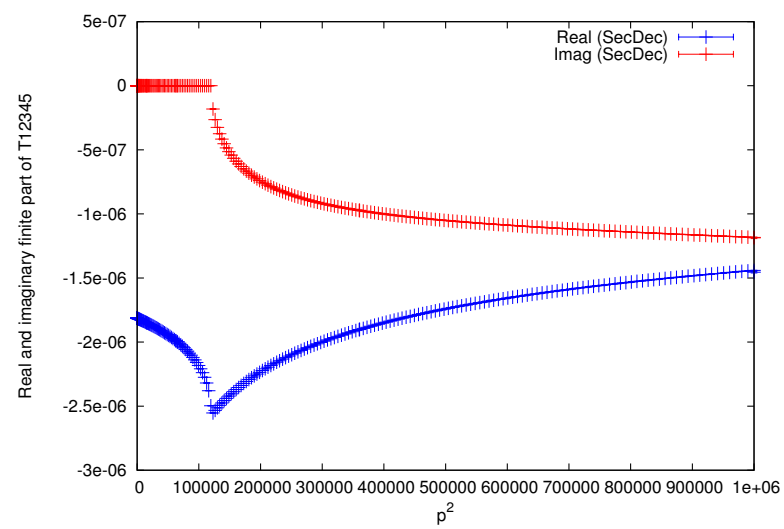

(b)

Figure 6: Numerical SECDEC results for the integrals of type (c) and (d) as defined in Fig. 5.

\section{Conclusions}

We have presented new features and applications of the program SECDEC, which can be used to calculate multi-loop integrals numerically in an automated way. Applications to non-planar twoloop master integrals occurring in $t \bar{t}$ production are shown, as well as the calculation of momentum dependent two-loop corrections to the masses of the neutral CP-even Higgs bosons in the MSSM. The program SECDEC is publicly available at http: / projects . hepforge. org/secdec.

\section{Acknowledgments}

We would like to thank Andreas von Manteuffel for comparisons with analytic results. We also thank Wolfgang Hollik for the productive collaboration on the calculation of the corrections to the MSSM Higgs masses and Sven Heinemeyer for interesting discussions. SB also wants to thank the organizers of RADCOR 2013 for the nice conference.

\section{References}

[1] Carter J and Heinrich G 2011 Comput.Phys.Commun. 182 1566-1581 (Preprint 1011.5493 )

[2] Borowka S, Carter J and Heinrich G 2013 Comput.Phys.Commun. 184 396-408 (Preprint $1204.4152)$

[3] Borowka S and Heinrich G 2013 Comput.Phys.Commun. 184 2552-2561 (Preprint 1303.1157 )

[4] Binoth T and Heinrich G 2000 Nucl. Phys. B585 741-759 (Preprint hep-ph / 0004013 )

[5] Roth M and Denner A 1996 Nucl. Phys. B479 495-514 (Preprint hep-ph / 9605420 )

[6] Hepp K 1966 Commun. Math. Phys. 2 301-326

[7] Smirnov A, Smirnov V and Tentyukov M 2011 Comput.Phys.Commun. 182 790-803 (Preprint $0912.0158)$

[8] Bogner C and Weinzierl S 2008 Comput. Phys. Commun. 178 596-610 (Preprint 0709.4092 )

[9] Gluza J, Kajda K, Riemann T and Yundin V 2011 Eur.Phys.J. C71 1516 (Preprint 1010.1667 ) 
[10] Heinrich G 2008 Int. J. Mod. Phys. A23 1457-1486 (Preprint 0803 . 4177)

[11] Hahn T 2005 Comput. Phys. Commun. 168 78-95 (Preprint hep-ph / 0404043 )

[12] von Manteuffel A and Studerus C 2013 (Preprint 1306.3504 )

[13] Borowka S and Heinrich G 2013 (Preprint 1309. 3492)

[14] Hempfling R and Hoang A H 1994 Phys.Lett. B331 99-106 (Preprint hep-ph/9401219)

[15] Heinemeyer S, Hollik W and Weiglein G 1998 Phys.Rev. D58 091701 (Preprint hep-ph/98032 77)

[16] Heinemeyer S, Hollik W and Weiglein G 1998 Phys.Lett. B440 296-304 (Preprint hep-ph/9807423)

[17] Heinemeyer S, Hollik W and Weiglein G 1999 Eur.Phys.J. C9 343-366 (Preprint hep-ph/9812472)

[18] Espinosa J R and Zhang R J 2000 JHEP 0003026 (Preprint hep-ph / 9912236)

[19] Degrassi G, Slavich P and Zwirner F 2001 Nucl.Phys. B611 403-422 (Preprint hep-ph/0105096)

[20] Espinosa J R and Zhang R J 2000 Nucl.Phys. B586 3-38 (Preprint hep-ph/ 0003246 )

[21] Brignole A, Degrassi G, Slavich P and Zwirner F 2002 Nucl.Phys. B631 195-218 (Preprint hep-ph/0112177)

[22] Brignole A, Degrassi G, Slavich P and Zwirner F 2002 Nucl.Phys. B643 79-92 (Preprint hep-ph/0206101)

[23] Dedes A, Degrassi G and Slavich P 2003 Nucl.Phys. B672 144-162 (Preprint hep-ph/0305127)

[24] Martin S P 2007 Phys.Rev. D75 055005 (Preprint hep-ph/ 0701051 )

[25] Harlander R, Kant P, Mihaila L and Steinhauser M 2008 Phys.Rev.Lett. 100191602 (Preprint $0803.0672)$

[26] Kant P, Harlander R, Mihaila L and Steinhauser M 2010 JHEP 1008104 (Preprint 1005.5709 )

[27] Martin S P 2003 Phys.Rev. D67 095012 (Preprint hep-ph/0211366)

[28] Martin S P 2005 Phys.Rev. D71 016012 (Preprint hep-ph/ 0405022 )

[29] Hahn T, Heinemeyer S, Hollik W, Rzehak H and Weiglein G 2009 Comput.Phys.Commun. 180 1426-1427

[30] Hahn T, Heinemeyer S, Hollik W, Rzehak H and Weiglein G 2010 Nucl.Phys.Proc.Suppl. 205-206 152-157 (Preprint 1007.0956$)$

[31] Weiglein G, Scharf R and Bohm M 1994 Nucl.Phys. B416 606-644 (Preprint hep-ph/9310358)

[32] Agrawal S, Hahn T and Mirabella E 2012 PoS LL2012 046 (Preprint 1210 . 2628)

[33] Borowka S et al. in preparation 